\title{
A REVERBERATION-BASED BLACK HOLE MASS FOR MCG-06-30-15
}

\author{
Misty C. Bentz ${ }^{1}$, Edward M. Cackett ${ }^{2}$, D. Michael Crenshaw ${ }^{1}$, Keith Horne $^{3}$, \\ Rachel StReET ${ }^{4}$, AND BenJamin OU-Yang ${ }^{1}$ \\ ${ }^{1}$ Department of Physics and Astronomy, Georgia State University, Atlanta, GA 30303, USA; bentz@astro.gsu.edu \\ ${ }^{2}$ Department of Physics and Astronomy, Wayne State University, 666 W. Hancock St., Detroit, MI 48201, USA \\ ${ }^{3}$ SUPA Physics and Astronomy, University of St. Andrews, Fife KY16 9SS, UK \\ ${ }^{4}$ Las Cumbres Observatory Global Telescope Network, 6740B Cortona Drive, Goleta, CA 93117, USA \\ Received 2016 June 24; revised 2016 August 1; accepted 2016 August 3; published 2016 October 18
}

\begin{abstract}
We present the results of a reverberation campaign targeting MGC-06-30-15. Spectrophotometric monitoring and broad-band photometric monitoring over the course of four months in spring 2012 allowed a determination of a time delay in the broad $\mathrm{H} \beta$ emission line of $\tau=5.3 \pm 1.8$ days in the rest frame of the active galactic nucleus (AGN). Combined with the width of the variable portion of the emission line, we determine a black hole mass of $M_{\mathrm{BH}}=(1.6 \pm 0.4) \times 10^{6} M_{\odot}$. Both the $\mathrm{H} \beta$ time delay and the black hole mass are in good agreement with expectations from the $R_{\mathrm{BLR}}-L$ and $M_{\mathrm{BH}}-\sigma_{\star}$ relationships for other reverberation-mapped AGNs. The $\mathrm{H} \beta$ time delay is also in good agreement with the relationship between $\mathrm{H} \beta$ and broad-band near-IR delays, in which the effective size of the broad-line region is $\sim 4-5$ times smaller than the inner edge of the dust torus. Additionally, the reverberation-based mass is in good agreement with estimates from the scaling relationship of the break in the X-ray power spectral density, and with constraints based on stellar kinematics derived from integral field spectroscopy of the inner $\sim 0.5 \mathrm{kpc}$ of the galaxy.
\end{abstract}

Key words: galaxies: active - galaxies: nuclei - galaxies: Seyfert

\section{INTRODUCTION}

It has been a century since Edward Fath (1913) first observed strong emission lines originating in the nucleus of NGC 1068 and discovered the first active galactic nucleus (AGN). Yet it is only in the last 30 years that AGNs have become synonymous with supermassive black holes (e.g., Rees 1984) and that supermassive black holes have become synonymous with galactic nuclei (e.g., Magorrian et al. 1998; Ferrarese \& Merritt 2000; Gebhardt et al. 2000; Ferrarese \& Ford 2005). Multiple independent lines of study focusing on a zoo of seemingly unrelated characteristics across the entire spectral energy distribution are now unified through our current understanding of the AGN phenomenon (e.g., Antonucci 1993; Urry \& Padovani 1995). The mass and the spin of the black hole, its only quantifiable characteristics, are two key parameters in our understanding of not only AGN physics (e.g., Krawczynski \& Treister 2013; Netzer 2015) but also galactic evolution (e.g., Fabian 2012; Kormendy \& Ho 2013; Heckman \& Best 2014; King \& Pounds 2015).

Astrophysical black holes can be characterized by their mass and spin, and being able to constrain both properties is rare. MCG-06-30-15 is one of only a handful of X-ray-bright AGNs where the $\mathrm{Fe} \mathrm{K} \alpha$ emission may be studied in detail, allowing a measure of the black hole spin. Tanaka et al. (1995) first detected a broad red wing of the $\mathrm{Fe} \mathrm{K} \alpha$ emission line, as is expected due to the strong gravitational redshift and relativistic Doppler effects from material in the innermost accretion disk. Relativistic reflection models fit to the $\mathrm{X}$-ray spectrum, including the $\mathrm{Fe} \mathrm{K} \alpha$ line, all indicate that the black hole spin is high, with a dimensionless spin parameter $a \gtrsim 0.9$ (Brenneman \& Reynolds 2006; Chiang \& Fabian 2011; Marinucci et al. 2014).

While the spin has been constrained for a decade now, the black hole mass $\left(M_{\mathrm{BH}}\right)$ of MCG-06-30-15 is less well known. Previous estimates of the mass have relied upon scaling relationships such as the break in the $\mathrm{X}$-ray power spectral density $\left(M_{\mathrm{BH}}=2.9_{-1.6}^{+1.8} \times 10^{6} M_{\odot}\right.$; McHardy et al. 2005) or the $M_{\mathrm{BH}}-\sigma_{\star}$ relationship $\left(M_{\mathrm{BH}}=(3-6) \times 10^{6} M_{\odot}\right.$; McHardy et al. 2005). Integral field spectroscopy of the inner $\sim 0.5 \mathrm{kpc}$ of the galaxy at high spatial resolution allowed Raimundo et al. (2013) to determine an upper limit on the black hole mass of $6 \times 10^{7} M_{\odot}$, but the integration time was somewhat short and precluded a stronger mass constraint.

Reverberation mapping (Blandford \& McKee 1982; Peterson 1993) is often employed for determining the black hole masses of AGNs of interest. Unlike dynamical modeling, which is limited by spatial resolution and therefore distance, reverberation mapping is applicable to all broad-lined AGNs regardless of location. The method makes use of the spectral variability of AGNs and determines the time delay between variations in the continuum emission (likely emitted from the accretion disk) and the response to these variations in the broad emission lines (emitted from the broad-line region (BLR)). The time delay is simply the responsivity-weighted average of the light travel time from the accretion disk to all of the BLR "clouds," and is generally interpreted as a measure of the average radius of the BLR for a specific emission species. In this case, the limiting resolution is temporal rather than spatial, and regions of the order of microarcseconds in size are routinely investigated (e.g., Peterson et al. 2002; Bentz et al. 2009b; Denney et al. 2010; Grier et al. 2012). The time delay combined with a measure of the velocity of the gas provides a constraint on the black hole mass through the virial theorem, modulo a scaling factor that accounts for the detailed geometry and kinematics of the line-emitting gas.

The requirements of dense temporal sampling and long monitoring baselines have generally limited reverberation campaigns to $1.0-4.0 \mathrm{~m}$ class telescopes in the past, and these have generally been located in the Northern Hemisphere. At a decl. of $\delta=-34^{\circ} .3$, MCG-06-30-15 has not been an ideal 

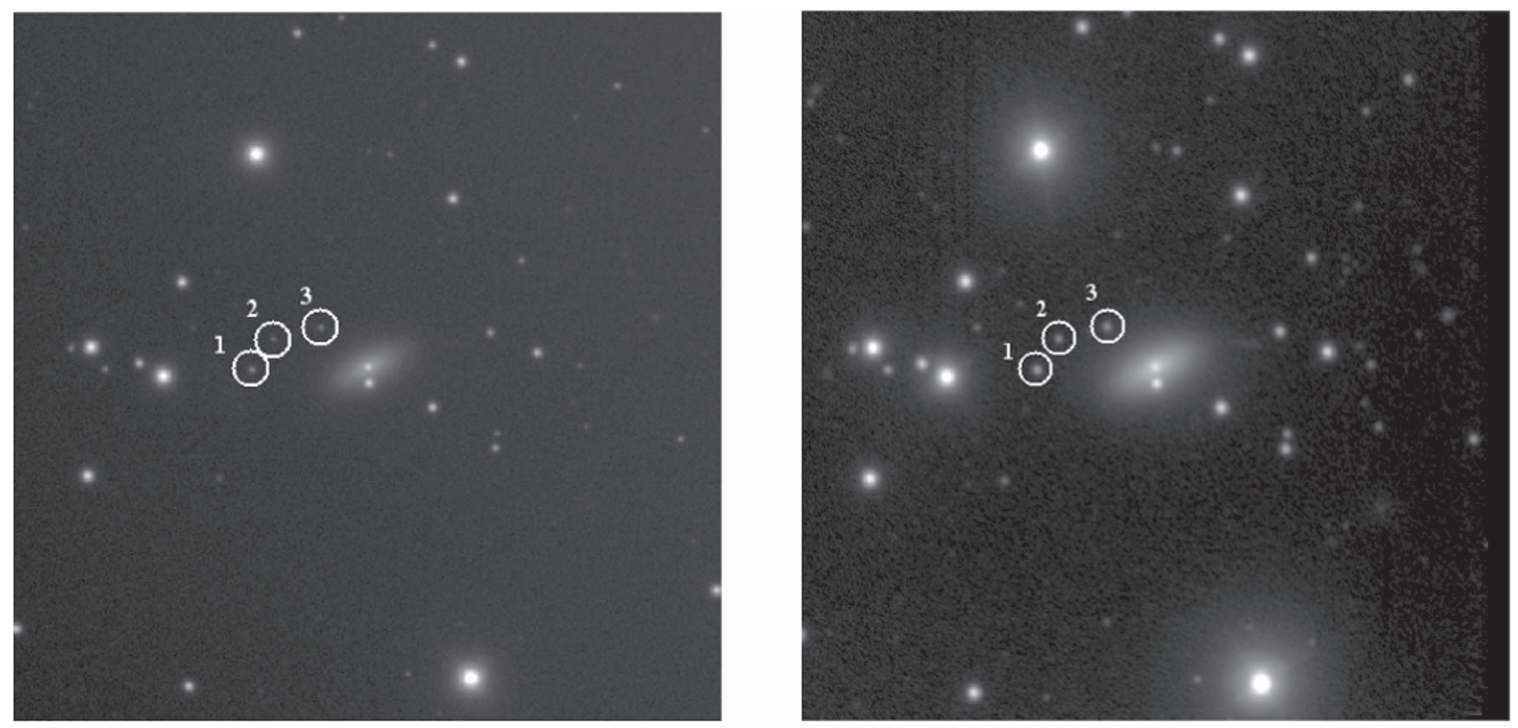

Figure 1. Reference images for the $V$-band FTS data set (left) and the $V$-band LT data set (right). The region displayed is $4^{\prime} \times 4^{\prime}$, with north up and east to the left. The average exposure time for the FTS images is $20 \mathrm{~s}$ at a typical airmass of 1.3, compared to $60 \mathrm{~s}$ exposures for the LT images with typical airmasses of 2.3. The marked field stars are common to the ground-based images and the high-resolution HST image and were used to determine the absolute flux scale of the FTS and LT images.

target for a reverberation campaign. Nevertheless, it was included in the set of AGNs monitored from the Lick Observatory as part of the LAMP 2008 program (Bentz et al. 2009b), but no time delays were detected because of the low level of variability of the source throughout the campaign combined with the non-optimal conditions under which it was observed each night (airmass $>3$ ).

We describe here the results of a reverberation mapping campaign for MCG-06-30-15 anchored by spectroscopy from the SMARTS $1.5 \mathrm{~m}$ telescope at Cerro Tololo Interamerican Observatory (CTIO). The variability of the target was somewhat increased during the monitoring period, compared to the 2008 campaign, and coupled with better data quality, we are able to determine a time delay for the broad $\mathrm{H} \beta$ emission line and a constraint on the black hole mass.

Throughout this paper, we adopt a $\Lambda$ CDM cosmology of $H_{0}=72 \mathrm{~km} \mathrm{~s}^{-1} \mathrm{Mpc}^{-1}, \Omega_{\mathrm{M}}=0.3, \Omega_{\Lambda}=0.7$.

\section{OBSERVATIONS}

For the monitoring campaign presented here, observations were carried out during spring 2012 with spectroscopy obtained at CTIO (latitude $=-30^{\circ}$ ), and photometry obtained at Siding Spring Observatory (latitude $=-31^{\circ}$ ) and at the Observatorio del Roque de los Muchachos at La Palma (latitude $=+28^{\circ}$ ). The details of each are described below.

\subsection{Photometry}

For reverberation mapping campaigns, photometric monitoring can provide a higher signal-to-noise ratio and better calibrated light curve of continuum variations than measurements taken directly from the spectra (e.g., Bentz et al. 2008, 2009b). In the cases of the $B$ and $V$ bands, especially, the contribution of broad-line emission to the bandpass is small compared to the continuum (e.g., Walsh et al. 2009). We therefore carried out broad-band $B$ and $V$ photometric monitoring at two sites to better constrain the continuum variations throughout our campaign: the $2 \mathrm{~m}$
Faulkes Telescope South (FTS) at Siding Spring Observatory and the $2 \mathrm{~m}$ Liverpool Telescope (LT) at the Observatorio del Roque de los Muchachos on La Palma in the Canary Islands.

Monitoring at FTS began on February 4 and continued through 2012 May 26 with the Spectral camera (UT dates here and throughout). Observations were obtained on 42 nights and generally consisted of $2 \times 35 \mathrm{~s}$ exposures in $B$ and $2 \times 20 \mathrm{~s}$ exposures in $V$ at an average airmass of 1.3. The field of view for the images was $10.5 \times 10.5$ with a pixel scale of 0 !" 304 in $2 \times 2$ binning mode.

Monitoring at LT utilized the RATCam and was carried out from 2012 February 20 through May 29. Observations were obtained on 42 nights at a typical airmass of 2.27 . Because of the higher expected airmass for these observations based on the latitude of the observatory relative to the decl. of the target, longer exposure times of $2 \times 60 \mathrm{~s}$ were utilized in $B$ and $V$. The field of view for RATCam is $4.6 \times 4 ! 6$, with a pixel scale of 0 ". 28 in $2 \times 2$ binning mode.

Both imaging data sets were analyzed through image subtraction methods in order to accurately constrain the nuclear variability of the galaxy. Images from a single observatory and a single filter were registered to a common alignment using the Sexterp routine (Siverd et al. 2012). We then employed the ISIS image subtraction package (Alard \& Lupton 1998; Alard 2000) to build a reference image (Figure 1) from the subset of images taken under the best conditions. The reference frame was convolved with a spatially varying kernel to match each individual image in the data set. Subtraction of the convolved reference from each image produces a residual image in which the components that are constant in flux have disappeared and only variable sources remain. Aperture photometry was then employed on the residual images to measure the amount of variable flux for the AGN. Analysis of these resultant light curves demonstrated that the $V$-band light curves exhibit the same features as the $B$-band light curves, but with less noise. We therefore focus our remaining analysis on the $V$-band light curves from our photometric monitoring. 


\subsection{Spectroscopy}

Spectroscopic monitoring was carried out with RCSpec on the SMARTS $1.5 \mathrm{~m}$ telescope at CTIO. Observations were scheduled to be carried out in queue-observing mode every other night during the period 2012 March 1-May 31. The spectrograph was equipped with the 600 line $\mathrm{mm}^{-1}$ blue grating (known as grating 26), giving a wavelength coverage of 3685-5400 $\AA$ and a nominal resolution of $1.5 \AA$ pix $^{-1}$ in the dispersion direction. Spectra were obtained through a $4^{\prime \prime}$ slit at a fixed position angle of $90^{\circ}$ (i.e., oriented east-west). The RCSpec detector, a Loral $1 \mathrm{~K}$ CCD, provides a spatial resolution of 1 !' $3 \mathrm{pix}^{-1}$.

Over the course of the campaign, spectra were obtained on 36 nights. Each visit consisted of two spectra with exposure times of $900 \mathrm{~s}$ that were obtained at an average airmass of 1.08. A spectrophotometric flux standard, LTT 4364, was also observed during each visit to assist with flux calibrations. Standard reductions were carried out with $\mathrm{IRAF}^{5}$, and an extraction width of 8 pixels (10!" 4) was adopted.

The initial flux calibration provided by the standard star is generally a good correction for the shape of the spectra, providing a useful way to remove the effects of the atmospheric transmission as well as the optics of the telescope and instrument. However, reverberation campaigns require high temporal sampling and therefore acquire spectra on all nights when the telescope may be safely used, often under nonphotometric conditions. We therefore require a method for carefully calibrating the overall flux level of each spectrum. This is generally accomplished by using the narrow emission lines as "internal" flux calibration sources, because the narrow lines do not vary on the timescales of a reverberation campaign. Specifically, we employ the spectral scaling method of van Groningen \& Wanders (1992) with the [O III] $\lambda \lambda 4959,5007$ emission lines as our internal calibration sources. The method minimizes the differences in a selected wavelength range between each individual spectrum and a reference spectrum created from a subset of the best data. It is therefore able to correct for slight differences in wavelength calibration, slight differences in resolution (caused by variable seeing and the employment of a wide spectroscopic slit), as well as differences in flux calibration. Peterson et al. (1998a) have shown that this method is able to provide relative spectrophotometry that is accurate to $\sim 2 \%$. To ensure the accuracy of our absolute spectrophotometry, we compared the integrated [O III] $\lambda 5007$ flux to published values determined from high-quality spectra observed under good conditions. We adopted a value of $f([\mathrm{O} \mathrm{III}])=1.0 \times 10^{-13} \mathrm{erg} \mathrm{s}^{-1} \mathrm{~cm}^{-1}$, in good agreement with Morris \& Ward (1988), Winkler (1992), and Reynolds et al. (1997).

The red lines in Figure 2 show the mean of all the calibrated spectra throughout the campaign (top) and the root mean square of the spectra (bottom), which highlights variable spectral components. It is immediately obvious that the variable (rms) spectrum is swamped by some combination of hostgalaxy light, possibly from mis-centering of the slit and poor seeing conditions, as well as scattered light from a nearby Milky Way star along the line of sight (5!"4 south of the nucleus and superimposed on the galactic disk). We therefore

\footnotetext{
5 IRAF is distributed by the National Optical Astronomy Observatory, which is operated by the Association of Universities for Research in Astronomy (AURA) under cooperative agreement with the National Science Foundation.
}

investigated a method for carefully subtracting the continuum of each spectrum, both the AGN power law and the starlight, through spectral modeling.

We employed the publicly available UlySS package (Koleva et al. 2009), which creates a linear combination of nonlinear model components convolved with a parametric lineof-sight velocity to match an observed spectrum. Our method started by modeling the mean spectrum of very high signal-tonoise ratio. We included a power-law component for the AGN continuum emission, multiple Gaussians for the emission lines (three components were necessary to match the $\mathrm{H} \beta$ profile), and a host-galaxy component parameterized by the Vazdekis models derived from the MILES library of empirical stellar spectra (Vazdekis et al. 2010). We make no attempt to interpret the best-fit parameters of our model, because our goal was simply to separate the line emission from the continuum components as cleanly as possible. We then held the number of model components and the age and metallicity of the best-fit Vazdekis model fixed, but allowed all other parameters to vary as we looped through all of the individual spectra of MCG-0630-15. In this way, we allow for variation of the power-law index, the relative contribution of power-law versus hostgalaxy starlight, and variability in the emission-line flux. Furthermore, we modeled the AGN spectra that had an initial flux calibration from a spectrophotometric standard star but had not yet been scaled with the code of van Groningen \& Wanders (1992) in order to get the best match between the models and the "untouched" observed spectra. The best-fit power-law and host-galaxy models were subtracted from each spectrum, providing a set of continuum-subtracted, pure emission-line spectra. In Figure 3, we show a typical example of a single spectrum, the best-fit continuum model (power law + starlight), and the resultant continuum-subtracted spectrum.

After modeling and subtraction of the continuum, all the spectra were then scaled with the method of van Groningen \& Wanders (1992) in the same way as previously described. The mean and rms of the scaled, continuum-subtracted spectra are shown by the black lines in Figure 2. The $\mathrm{H} \beta$ emission line, though weak, is apparent in the continuum-subtracted rms spectrum.

\section{ANALYSIS OF THE LIGHT CURVE}

Emission-line light curves were determined from both the original, scaled spectra and the continuum-subtracted, scaled spectra, in order to verify that our continuum subtraction method did not introduce artificial variability. In both cases, a local linear continuum was fit underneath the emission line, and the flux above the continuum was integrated. We included this local continuum fit even for the continuum-subtracted spectra to ensure that any small mismatches between the model continuum and that of the spectrum were accounted for and removed from the emission-line measurements. Multiple measurements from a single night were then averaged together to decrease the noise in the resultant light curves, which are displayed in Figure 4. The $\mathrm{H} \beta$ light curve derived from the scaled, continuum-subtracted spectra (black points) matches extremely well the $\mathrm{H} \beta$ light curve derived from the scaled-only spectra (red points). The two light curves are virtually identical, with the most obvious difference being a slight offset in which the continuum-subtracted spectra have an elevated $\mathrm{H} \beta$ flux (due to correction of the intrinsic $\mathrm{H} \beta$ absorption from the starlight). A linear fit to the fluxes determined from each method shows 


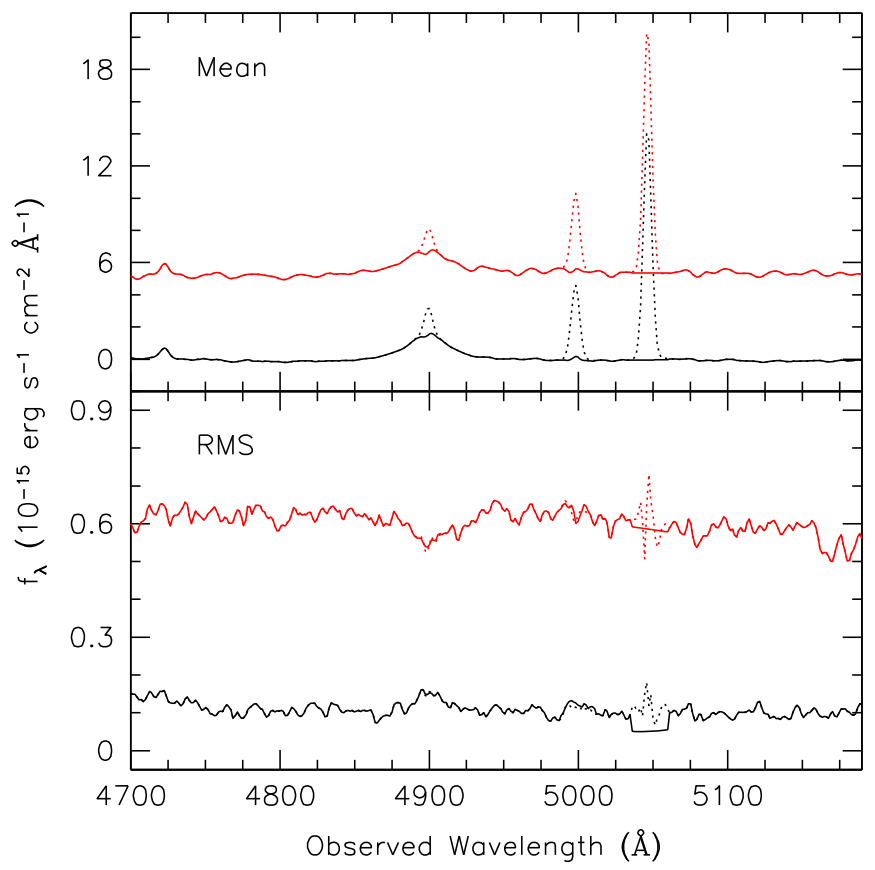

Figure 2. Top: mean spectrum of MCG-06-30-15 (red line) and the mean spectrum created after subtraction of the continuum from each individual spectrum (black line). The dotted lines show the narrow component of $\mathrm{H} \beta$ and the [O III] $\lambda \lambda 4959,5007$ doublet. Bottom: rms of the original calibrated spectra (red line) and rms of the continuum-subtracted spectra (black line). The variable portion of the $\mathrm{H} \beta$ emission line is apparent once the strongly variable continuum has been removed.

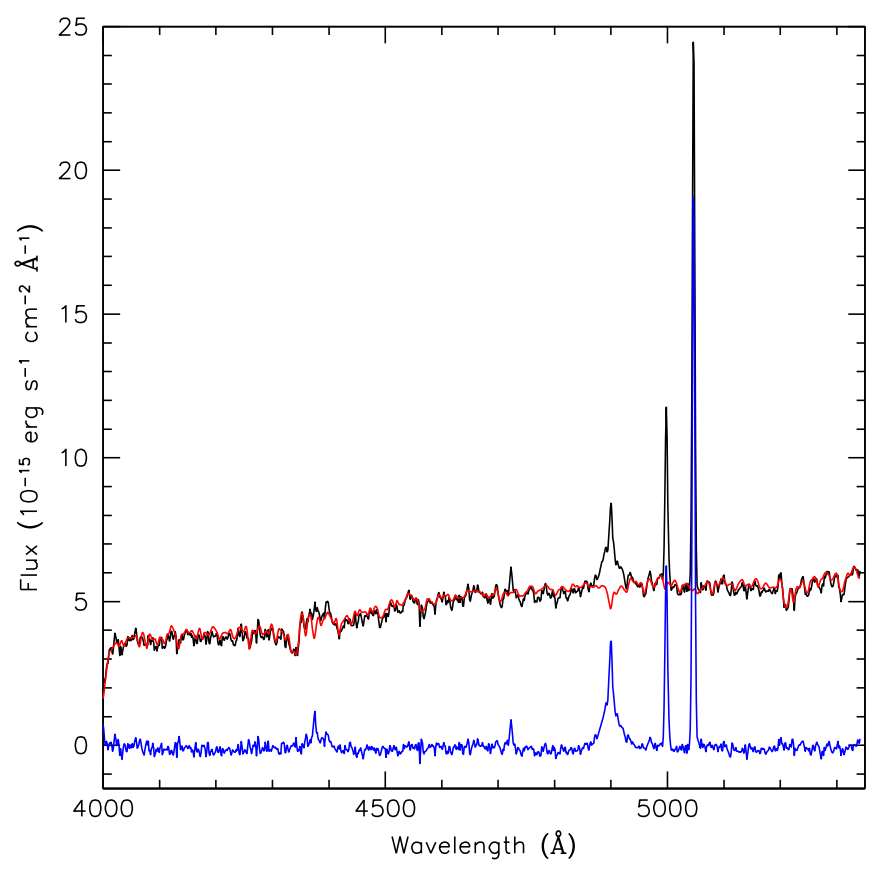

Figure 3. The black line displays a spectrum of MCG-06-30-15 taken on the night of 2012 March 1, with the best-fit continuum model overplotted in red. The continuum model comprises a power-law component and a model hostgalaxy component. The continuum-subtracted spectrum is plotted in blue.

that the difference between the two light curves is almost entirely a simple offset, with very minimal flux dependence (close to a slope of 1).

The differential light curves derived from image subtraction analysis of the $V$-band photometry were converted to absolute flux units in the following way. First, the reference image for each set of observations was modeled with the two-dimensional surface brightness fitting program GALFIT. The shape parameters of the galaxy bulge and disk were matched to those derived from the analysis of a high-resolution, medium- $V$ Hubble Space Telescope (HST) image (see Section 6.1). Field stars common to both the HST image and the ground-based images were also modeled (circled in Figure 1), and the magnitudes of the field stars derived from the HST image were used to set the absolute flux calibration of the ground-based images. The brightness of the AGN point-spread function (PSF) in each ground-based reference image was then added back to the differential flux derived from the image subtraction analysis for that set of photometry. While the overall flux scale of the light curve is not important, we found a slight offset of 0.2 mag between the FTS- and the LT-calibrated photometry, so we adjusted the LT photometry to match that of the FTS, since the FTS observations were generally obtained under better conditions. The calibrated photometric light curves were then combined together and measurements coincident within 0.5 days were averaged together. The final $V$-band light curve is displayed in the top left panel of Figure 4.

Table 2 gives the variability statistics for the final $V$-band and $\mathrm{H} \beta$ emission-line light curves displayed in Figure 4. Column (1) lists the spectral feature and column (2) gives the number of measurements in the light curve. Columns (3) and (4) list the average and median time separation between measurements, respectively. Column (5) gives the mean flux and standard deviation of the light curve, and column (6) lists the mean fractional error (based on the comparison of observations that are closely spaced in time). Column (7) lists the amplitude of fractional rms variability, computed as

$$
F_{\mathrm{var}}=\frac{\sqrt{\sigma^{2}-\delta^{2}}}{\langle F\rangle}
$$

where $\sigma^{2}$ is the variance of the fluxes, $\delta^{2}$ is their mean square uncertainty, and $\langle F\rangle$ is the mean flux (Rodríguez-Pascual et al. 1997). The uncertainty on $F_{\text {var }}$ is quantified as

$$
\sigma_{F_{\mathrm{var}}}=\frac{1}{F_{\mathrm{var}}} \sqrt{\frac{1}{2 N}} \frac{\sigma^{2}}{\langle F\rangle^{2}}
$$

(Edelson et al. 2002). Column (8) is the ratio of the maximum to the minimum flux in the light curve. At first glance, the $F_{\text {var }}$ values for the $\mathrm{H} \beta$ light curves from the continuum-subtracted (CS) and the unsubtracted spectra appear quite discrepant given the similarities in the light curves. The disagreement arises solely due to two data points in each light curve with large uncertainties, reflecting the marginal conditions under which the observations were obtained. Removal of those two data points from each light curve modifies the $F_{\text {var }}$ value for the continuum-subtracted spectra only slightly, increasing it from $0.075 \pm 0.011$ to $0.080 \pm 0.010$. The $F_{\text {var }}$ value for the unsubtracted spectra, however, decreases significantly from $0.132 \pm 0.018$ to $0.099 \pm 0.012$, bringing the $F_{\text {var }}$ values for the two light curves into better agreement.

To determine the mean time delay of the $\mathrm{H} \beta$ emission line relative to the continuum variations, we cross-correlated the $\mathrm{H} \beta$ light curve derived from the continuum-subtracted spectra (Figure 4, black points) with the $V$-band light curve (both tabulated in Table 1). We employed the interpolated cross- 


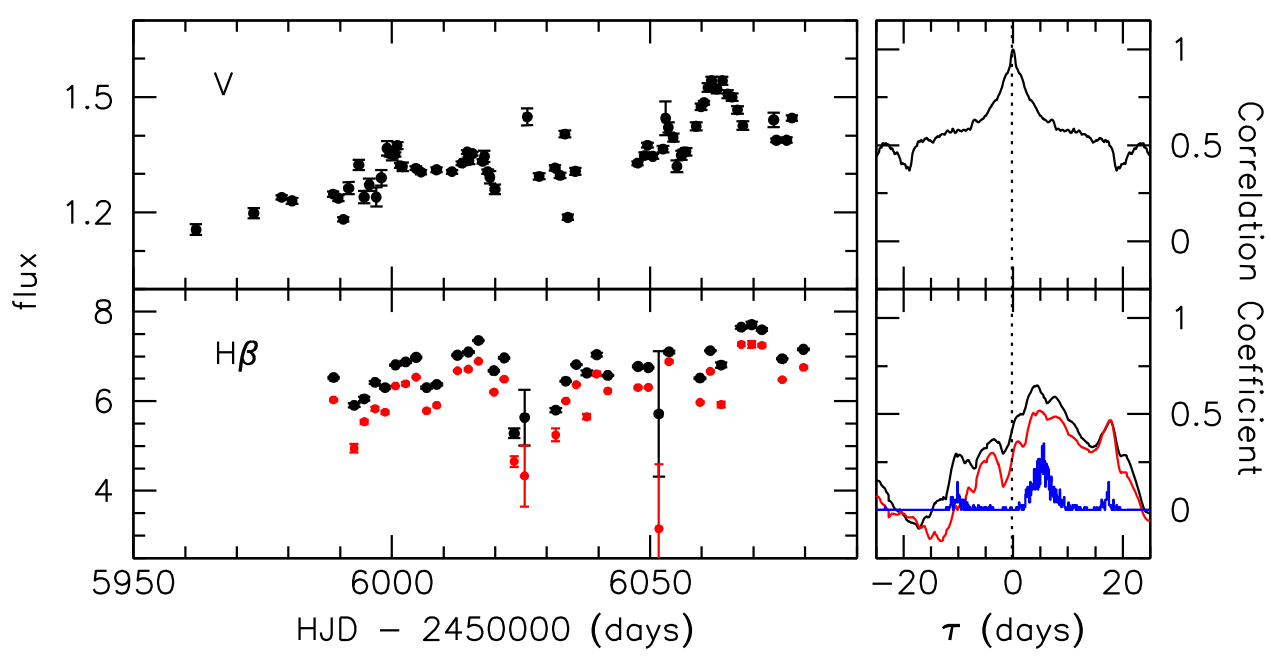

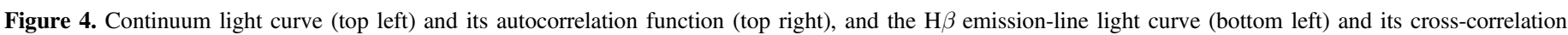

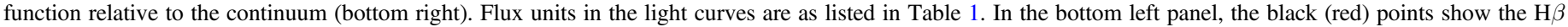

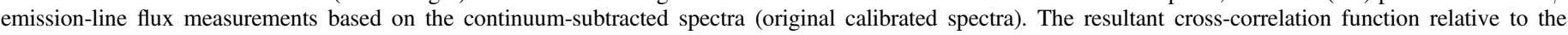

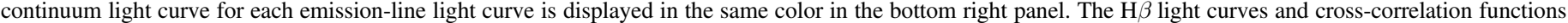

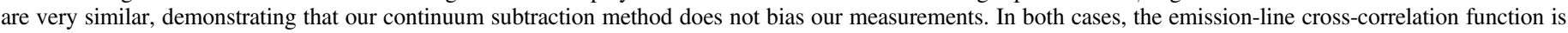

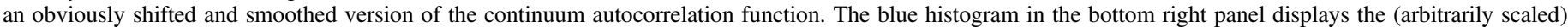

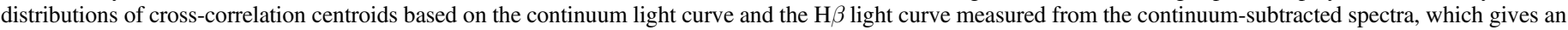
$\mathrm{H} \beta$ time delay of $\tau=5.4 \pm 1.8$ days.

correlation function (ICCF) method (Gaskell \& Sparke 1986; Gaskell \& Peterson 1987) with the modifications of White \& Peterson (1994). This method determines the cross-correlation function (CCF) twice, first by interpolating the continuum light curve and then by interpolating the emission-line light curve in the second pass. The resultant CCF, which is the average of the two, is shown by the black solid line in the bottom right panel of Figure 4. For reference, we calculated the autocorrelation function of the $V$-band light curve, shown as the solid line in the top right panel of Figure 4. Also displayed in Figure 4 is the $\mathrm{CCF}$ for the $\mathrm{H} \beta$ light curve derived from the original, scaled spectra (red points) compared to the $V$ band (red line). As expected, given the nearly identical variations in the $\mathrm{H} \beta$ light curves, the CCFs of the two relative to $V$ are also nearly identical. However, the slightly reduced noise in the $\mathrm{H} \beta$ light curve derived from the continuum-subtracted spectra provides a higher correlation coefficient at the preferred time delay. We therefore focus the remainder of our analysis on the $\mathrm{H} \beta$ light curve derived from the continuum-subtracted spectra.

CCFs can be characterized by their maximum value $\left(r_{\max }\right)$, the time delay at which the CCF maximum occurs $\left(\tau_{\text {peak }}\right)$, and the centroid of the points near the peak $\left(\tau_{\text {cent }}\right)$ above a threshold value of $0.8 r_{\max }$. However, a single CCF does not provide any information on the uncertainties inherent in these measurements. We therefore employ the "flux randomization/random subset sampling" (FR/RSS) method of Peterson et al. (1998b, 2004), which is a Monte Carlo approach for determining the uncertainties in our measured time delays. For a sample of $N$ data points, a selection of $N$ points is chosen without regard to whether a datum was previously chosen or not. The typical number of points that is not sampled in a single realization is $\sim 1 / e$. A point that is sampled $1 \leqslant n \leqslant N$ times has its uncertainty reduced by a factor of $n^{1 / 2}$. This "random subset sampling" step is therefore able to assess the uncertainty in the time delay that arises from an individual data point in the light curve. The "flux randomization" step takes each of the selected points and modifies the flux value by a Gaussian deviation of the uncertainty. In this way, the effect of the measurement uncertainties on the recovered time delay is also assessed. The final modified light curves are then cross-correlated with the ICCF method described above, and the values of $r_{\text {max }}, \tau_{\text {peak }}$, and $\tau_{\text {cent }}$ are recorded. The entire process is repeated 1000 times, and distributions of these values are built up from all of the realizations. We take the medians of the cross-correlation peak distribution and the cross-correlation centroid distribution (CCCD) as $\tau_{\text {peak }}$ and $\tau_{\text {cent }}$, respectively. The uncertainties on these values are quoted so that $15.87 \%$ of the realizations fall above and $15.87 \%$ fall below the range of uncertainties, corresponding to $\pm 1 \sigma$ for a Gaussian distribution. The final measurements are quoted in Table 3 in both the observer's frame and the rest frame of the AGN, and the CCCD is displayed in the bottom right panel of Figure 4 as the blue histogram (arbitrarily scaled). The mean of the distribution agrees well with the time delay inferred from the CCF.

For comparison, we also determined the $\mathrm{H} \beta$ time delay using the JAVELIN code ( $\mathrm{Zu}$ et al. 2011). JAVELIN employs a damped random walk to model the continuum variations, and then determines the best reprocessing model by quantifying the shifting and smoothing parameters necessary to reproduce the emission-line light curve (see Figure 5). The uncertainties on the model parameters are assessed through a Bayesian Markov chain Monte Carlo (MCMC) method. We include the JAVELIN time delay as $\tau_{\text {jav }}$ in Table 3 .

Additionally, we used a Markov chain Monte Carlo code MCMCRev to fit a linearized echo model to the $V$-band and $\mathrm{H} \beta$ light curves (see Figure 6). This models the $V$-band light curve with a Fourier series constrained by the light-curve data and with a random-walk prior that mimics typical AGN continuum variations on $1-100$ day timescales. $\mathrm{H} \beta$ variations are modeled as an echo of those in the $V$ band. A two-parameter delay distribution, specifically

$$
\Psi(\tau)=\frac{\Psi_{0}}{2 \tau_{0}} \frac{\left(\tau / \tau_{0}\right)^{9 / 4}}{\cosh \left(\tau / \tau_{0}\right)-1},
$$


Table 1

$V$-band and $\mathrm{H} \beta$ Light Curves

\begin{tabular}{|c|c|c|c|}
\hline $\begin{array}{l}\text { HJD } \\
(-2450000)\end{array}$ & $\begin{array}{c}f_{\lambda}(V) \\
\left(10^{-15} \mathrm{erg} \mathrm{s}^{-1} \mathrm{~cm}^{-2} \AA^{-1}\right)\end{array}$ & $\begin{array}{c}\text { HJD } \\
(-2450000)\end{array}$ & $\begin{array}{c}f(\mathrm{H} \beta) \\
\left(10^{-15} \mathrm{erg} \mathrm{s}^{-1} \mathrm{~cm}^{-2}\right)\end{array}$ \\
\hline 5962.0886 & $1.155 \pm 0.014$ & 5988.7106 & $65.27 \pm 0.19$ \\
\hline 5973.2684 & $1.198 \pm 0.013$ & 5992.7187 & $59.09 \pm 0.43$ \\
\hline 5978.6867 & $1.239 \pm 0.006$ & 5994.6647 & $60.54 \pm 0.44$ \\
\hline 5980.6836 & $1.230 \pm 0.008$ & 5996.7522 & $64.18 \pm 0.34$ \\
\hline 5988.6514 & $1.248 \pm 0.007$ & 5998.6879 & $63.05 \pm 0.31$ \\
\hline 5989.6408 & $1.237 \pm 0.008$ & 6000.7037 & $68.11 \pm 0.12$ \\
\hline 5990.6260 & $1.182 \pm 0.006$ & 6002.6755 & $68.78 \pm 0.28$ \\
\hline 5991.6260 & $1.263 \pm 0.016$ & 6004.6979 & $69.77 \pm 0.16$ \\
\hline 5993.6192 & $1.324 \pm 0.013$ & 6006.7308 & $63.05 \pm 0.21$ \\
\hline 5994.6396 & $1.240 \pm 0.016$ & 6008.6991 & $63.75 \pm 0.22$ \\
\hline 5995.6130 & $1.272 \pm 0.016$ & 6012.7055 & $70.27 \pm 0.17$ \\
\hline 5996.9981 & $1.240 \pm 0.025$ & 6014.8060 & $70.97 \pm 0.22$ \\
\hline 5997.9778 & $1.290 \pm 0.020$ & 6016.7763 & $73.57 \pm 0.13$ \\
\hline 5999.0720 & $1.367 \pm 0.019$ & 6019.7684 & $66.81 \pm 0.17$ \\
\hline 5999.6167 & $1.353 \pm 0.007$ & 6021.7506 & $69.67 \pm 0.15$ \\
\hline 6000.0813 & $1.353 \pm 0.017$ & 6023.6823 & $52.83 \pm 1.06$ \\
\hline 6000.6030 & $1.351 \pm 0.006$ & 6025.6963 & $56.35 \pm 6.20$ \\
\hline 6001.0505 & $1.374 \pm 0.009$ & 6031.7218 & $58.02 \pm 0.48$ \\
\hline 6001.6535 & $1.322 \pm 0.006$ & 6033.6779 & $64.49 \pm 0.13$ \\
\hline 6002.0935 & $1.319 \pm 0.011$ & 6035.6990 & $68.14 \pm 0.11$ \\
\hline 6004.6577 & $1.315 \pm 0.005$ & 6037.7316 & $66.33 \pm 0.53$ \\
\hline 6005.6684 & $1.305 \pm 0.006$ & 6039.6459 & $70.39 \pm 0.39$ \\
\hline 6008.6396 & $1.311 \pm 0.006$ & 6041.8089 & $65.73 \pm 0.14$ \\
\hline 6011.6284 & $1.306 \pm 0.005$ & 6047.6504 & $67.73 \pm 0.17$ \\
\hline 6013.5708 & $1.328 \pm 0.005$ & 6049.6533 & $67.52 \pm 0.14$ \\
\hline 6014.5952 & $1.357 \pm 0.005$ & 6051.6786 & $57.17 \pm 14.03$ \\
\hline 6014.9191 & $1.335 \pm 0.009$ & 6053.6594 & $70.99 \pm 0.29$ \\
\hline 6015.5847 & $1.353 \pm 0.005$ & 6059.6553 & $65.15 \pm 0.17$ \\
\hline 6017.5606 & $1.333 \pm 0.006$ & 6061.6300 & $71.26 \pm 0.14$ \\
\hline 6017.9114 & $1.346 \pm 0.015$ & 6063.7588 & $68.08 \pm 0.56$ \\
\hline 6018.5535 & $1.306 \pm 0.006$ & 6067.6406 & $76.50 \pm 0.29$ \\
\hline 6018.9996 & $1.291 \pm 0.016$ & 6069.6631 & $77.08 \pm 0.68$ \\
\hline 6019.9486 & $1.260 \pm 0.012$ & 6071.6367 & $75.97 \pm 0.28$ \\
\hline 6026.2009 & $1.449 \pm 0.022$ & 6075.5645 & $69.45 \pm 0.14$ \\
\hline 6028.5347 & $1.294 \pm 0.008$ & 6079.6835 & $71.57 \pm 0.17$ \\
\hline 6031.5757 & $1.316 \pm 0.007$ & $\ldots$ & $\ldots$ \\
\hline 6032.5654 & $1.296 \pm 0.007$ & $\cdots$ & $\cdots$ \\
\hline 6033.5161 & $1.404 \pm 0.009$ & $\ldots$ & $\ldots$ \\
\hline 6034.0558 & $1.187 \pm 0.007$ & $\ldots$ & $\ldots$ \\
\hline 6035.5098 & $1.307 \pm 0.009$ & $\ldots$ & $\ldots$ \\
\hline 6047.5410 & $1.328 \pm 0.005$ & $\ldots$ & $\ldots$ \\
\hline 6048.8850 & $1.348 \pm 0.009$ & $\cdots$ & $\cdots$ \\
\hline 6049.4712 & $1.375 \pm 0.006$ & $\cdots$ & $\cdots$ \\
\hline 6050.5168 & $1.346 \pm 0.006$ & $\ldots$ & $\ldots$ \\
\hline 6052.4839 & $1.365 \pm 0.008$ & $\ldots$ & $\ldots$ \\
\hline 6052.9778 & $1.445 \pm 0.044$ & $\cdots$ & $\cdots$ \\
\hline 6053.5425 & $1.421 \pm 0.013$ & $\cdots$ & $\cdots$ \\
\hline 6054.4863 & $1.395 \pm 0.010$ & $\ldots$ & $\ldots$ \\
\hline 6055.1726 & $1.320 \pm 0.015$ & $\cdots$ & $\cdots$ \\
\hline 6056.0708 & $1.349 \pm 0.012$ & $\ldots$ & $\cdots$ \\
\hline 6056.4541 & $1.357 \pm 0.006$ & $\ldots$ & $\ldots$ \\
\hline 6056.9783 & $1.358 \pm 0.010$ & $\ldots$ & $\ldots$ \\
\hline 6058.9017 & $1.424 \pm 0.010$ & $\ldots$ & $\ldots$ \\
\hline 6059.8591 & $1.475 \pm 0.008$ & $\cdots$ & $\cdots$ \\
\hline 6060.4424 & $1.486 \pm 0.005$ & $\cdots$ & $\cdots$ \\
\hline 6061.1837 & $1.525 \pm 0.011$ & $\ldots$ & $\ldots$ \\
\hline 6061.8630 & $1.543 \pm 0.010$ & $\ldots$ & $\ldots$ \\
\hline 6062.9246 & $1.521 \pm 0.011$ & $\cdots$ & $\cdots$ \\
\hline 6063.9783 & $1.543 \pm 0.010$ & $\cdots$ & $\cdots$ \\
\hline 6065.0056 & $1.508 \pm 0.011$ & $\cdots$ & $\cdots$ \\
\hline 6065.8806 & $1.500 \pm 0.010$ & $\cdots$ & $\cdots$ \\
\hline 6066.8920 & $1.467 \pm 0.010$ & $\ldots$ & $\cdots$ \\
\hline 6067.9285 & $1.426 \pm 0.011$ & $\ldots$ & $\ldots$ \\
\hline
\end{tabular}


Table 1

(Continued)

\begin{tabular}{lccc}
\hline \hline $\begin{array}{l}\text { HJD } \\
(-2450000)\end{array}$ & $\begin{array}{c}f_{\lambda}(V) \\
\left(10^{-15} \mathrm{erg} \mathrm{s}^{-1} \mathrm{~cm}^{-2} \AA^{-1}\right)\end{array}$ & $\begin{array}{c}\text { HJD } \\
(-2450000)\end{array}$ & \\
\hline 6073.9258 & $1.441 \pm 0.019$ & $\cdots$ & $f(\mathrm{H} \beta)$ \\
6074.4324 & $1.388 \pm 0.005$ & $\cdots$ & $\cdots$ \\
6076.4253 & $1.388 \pm 0.007$ & $\cdots$ & $\cdots$ \\
6077.4556 & $1.446 \pm 0.007$ & $\cdots$ & $\cdots$ \\
\hline
\end{tabular}

Table 2

Light-curve Statistics

\begin{tabular}{lcccccr}
\hline \hline Time Series & $N$ & $\begin{array}{c}\langle T\rangle \\
\text { (days) }\end{array}$ & $\begin{array}{c}T_{\text {median }} \\
\text { (days) }\end{array}$ & $\langle F\rangle^{\mathrm{a}}$ & $\left\langle\sigma_{F} / F\right\rangle$ & $F_{\text {var }}$ \\
\hline $\mathrm{V}$ & 67 & $1.75 \pm 2.28$ & 1.00 & $1.35 \pm 0.09$ & 0.008 & $0.066 \pm 0.006$ \\
$\mathrm{H} \beta$, non-CS & 35 & $2.68 \pm 1.26$ & 2.02 & $6.10 \pm 0.85$ & 0.023 & $0.132 \pm 0.018$ \\
$\mathrm{H} \beta$, CS & 35 & $2.68 \pm 1.26$ & 2.02 & $6.68 \pm 0.57$ & 0.014 & $0.075 \pm 0.011$ \\
\hline
\end{tabular}

Note.

${ }^{\text {a }} V$-band flux density is in units of $10^{-15} \mathrm{erg} \mathrm{s}^{-1} \mathrm{~cm}^{-2} \AA^{-1}$ and $\mathrm{H} \beta$ flux is in units of $10^{-14} \mathrm{erg} \mathrm{s}^{-1} \mathrm{~cm}^{-2}$.

Table 3

Time Lags and Line Widths

\begin{tabular}{lccccc}
\hline \hline Frame & $\begin{array}{c}\tau_{\text {cent }} \\
(\text { days })\end{array}$ & $\begin{array}{c}\tau_{\text {peak }} \\
(\text { days })\end{array}$ & $\begin{array}{c}\tau_{\text {jav }} \\
(\text { days })\end{array}$ & Spectrum & $\begin{array}{c}\text { FWHM } \\
\left(\mathrm{km} \mathrm{s}^{-1}\right)\end{array}$ \\
\hline Observed & $5.37_{-1.76}^{+1.87}$ & $4.40_{-0.80}^{+3.10}$ & $4.40_{-0.10}^{+0.08}$ & Mean & $1958.4 \pm 74.8$ \\
Rest frame & $5.33_{-1.75}^{+1.86}$ & $4.37_{-0.79}^{+3.08}$ & $4.37_{-0.10}^{+0.08}$ & rms & $1422.0 \pm 416.4$ \\
\hline
\end{tabular}

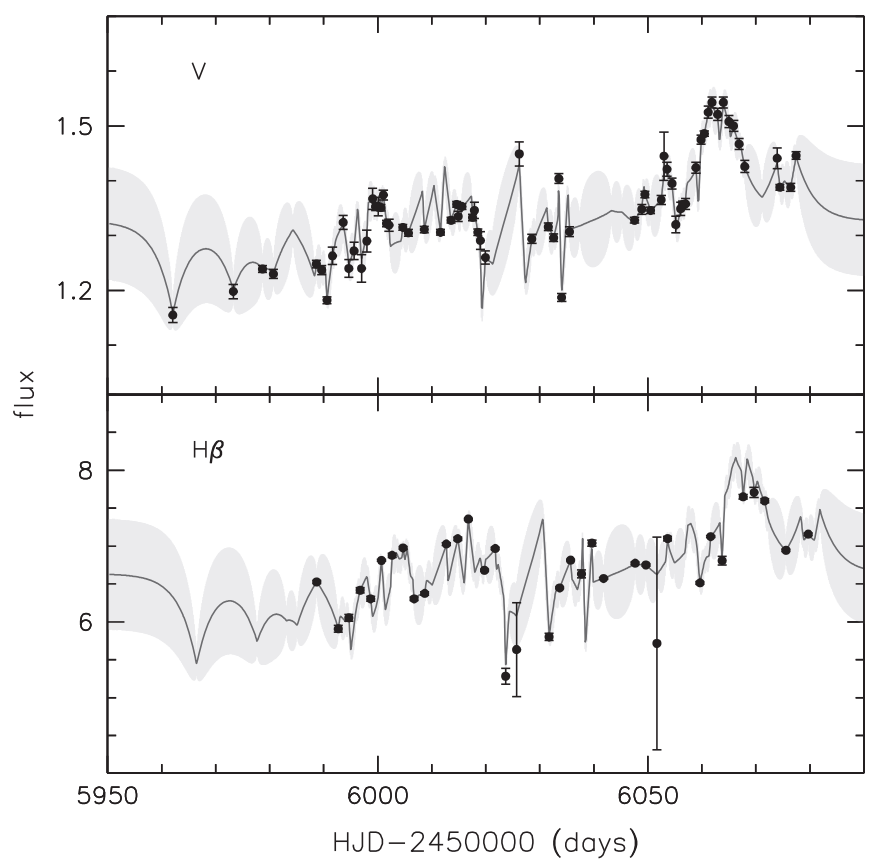

Figure 5. $V$-band light curve (data points, top) and $\mathrm{H} \beta$ light curve derived from the continuum-subtracted spectra (data points, bottom). The mean JAVELIN models and uncertainties are overplotted as the solid lines and gray shaded regions, respectively. The model uncertainties are derived from the standard deviation of the individual realizations. JAVELIN finds a best-fit $\mathrm{H} \beta$ time delay of $4.4 \pm 0.1$ days.

enforces causality $(\tau>0)$ and has a width proportional to the mean delay $\langle\tau\rangle \approx 5 \tau_{0}$. Three further parameters are the mean $\mathrm{H} \beta$ flux, and two factors that scale the nominal $\mathrm{H} \beta$ and $\mathrm{V}$-band error bars. The mean and rms of the MCMC samples give the $\mathrm{H} \beta$ delay as $\langle\tau\rangle=4.6 \pm 2.8$ days, in agreement with the other techniques.

\section{LINE WIDTH MEASUREMENTS}

The broad emission lines in AGN spectra are interpreted as being Doppler-broadened through the bulk gas motions deep in the potential well of the black hole. Therefore, the width of the broad line is a constraint on the line-of-sight velocity of the gas. The narrow emission lines, however, are produced by gas that is well outside the nucleus of the AGN and does not reverberate on the timescales of a few months. It is therefore important that we remove the narrow contribution to the $\mathrm{H} \beta$ emission line before attempting to measure the line width.

We accomplish this by using the [O III] $\lambda 5007$ emission line as a template for the narrow emission lines in the spectrum. The template is shifted and scaled by an appropriate amount to account for both the [O III] $\lambda 4959$ line and the $\mathrm{H} \beta$ narrow line. We adopted a scale factor of $\lambda 4959 / \lambda 5007=0.34$ (Storey $\&$ Zeippen 2000) and, through trial and error, determined a scale factor of $\mathrm{H} \beta / \lambda 5007=0.10$. The original and narrow-linesubtracted spectra are displayed in Figure 2.

From the narrow-line-subtracted spectra, we determined the emission line width in both the mean and rms of the continuum-subtracted spectra. A local linear continuum was determined from two continuum windows on either side of the emission line, and the width was determined directly from the measurements above this local continuum. We report the line width as the full width at half the maximum flux (FWHM) and also as the second moment of the line profile, or the line dispersion, $\sigma_{\text {line. }}$. 


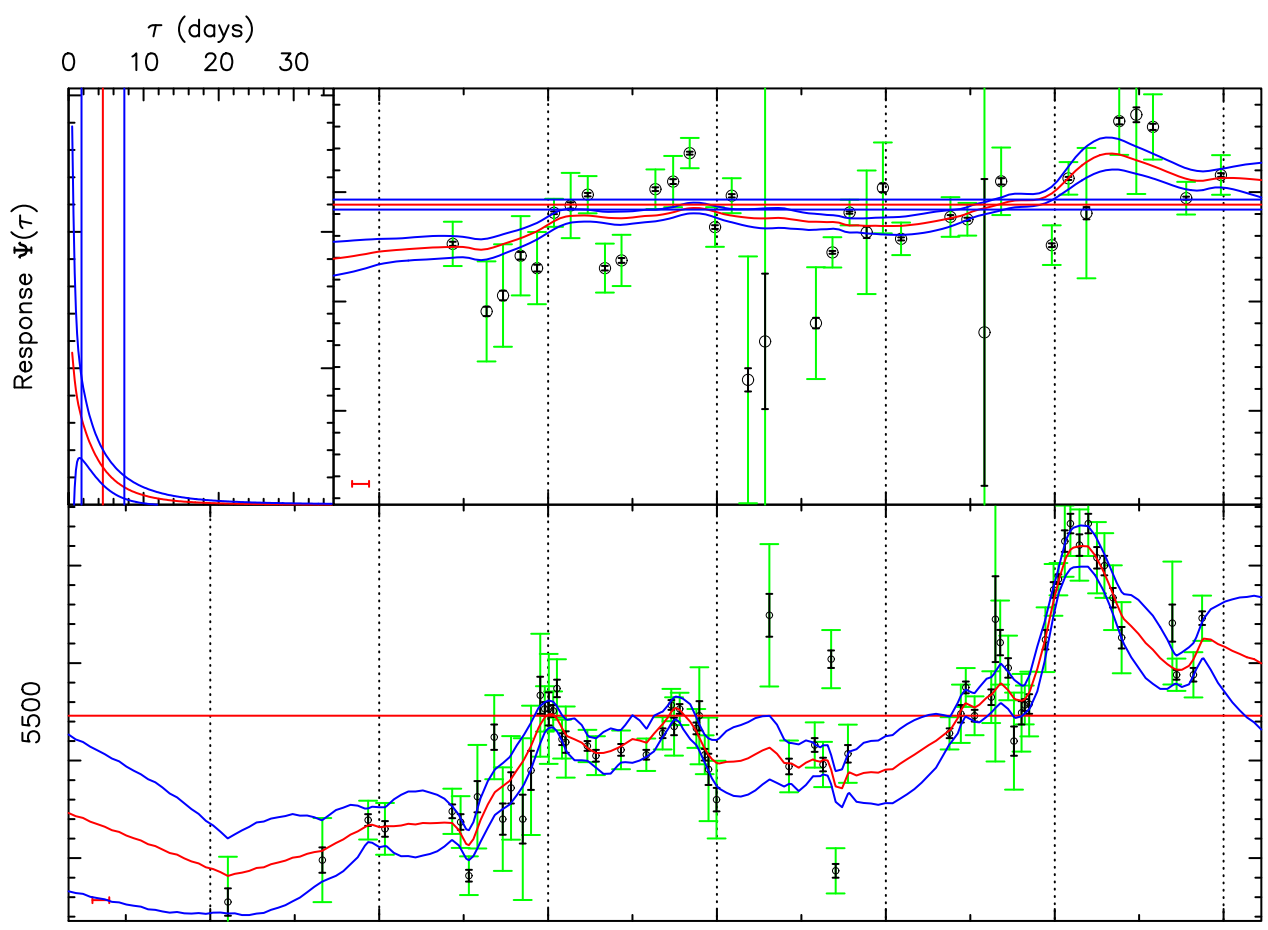

Figure 6. Results of the MCMCRev fit of a linearized echo model to the $\mathrm{H} \beta$ (top) and $V$-band (bottom) light-curve data. The red and blue curves give the mean \pm rms over MCMC samples of the model light curves and the delay distribution (top left). The mean and rms of MCMC samples give the mean $\mathrm{H} \beta$ delay as $\langle\tau\rangle=4.6 \pm 2.8$ days.

The uncertainties on the line width measurements were determined from a Monte Carlo random subset sampling method. For a set of $N$ spectra, we select $N$ without regard to whether a spectrum was previously chosen or not. The mean and rms of this subset are determined, and the FWHM and $\sigma_{\text {line }}$ are tabulated. The process is repeated 1000 times, and a distribution of each measurement is built up. In this way, the effect of any particular spectrum on the line width measurements is assessed. We also included a slight modification in which the continuum windows on either side of the emission line were allowed to vary in size and exact placement within an acceptable range, thereby assessing the effect of the choice of continuum window on the final measurements. This modification generally has little or no effect on the line widths derived from the rms spectrum, where noise already dominates the uncertainties, but it slightly increases the uncertainties on the line widths derived from the mean spectrum (Bentz et al. 2009b). The mean and standard deviation of each distribution are adopted as the measurement value and its uncertainty, respectively.

Finally, we also corrected for the dispersion of the spectrograph following the method employed by Peterson et al. (2004), in which the observed line width can be described as a combination of the intrinsic line width, $\Delta \lambda_{\text {true }}$, and the spectrograph dispersion, $\Delta \lambda_{\text {disp }}$, such that

$$
\Delta \lambda_{\text {obs }}^{2} \approx \Delta \lambda_{\text {true }}^{2}+\Delta \lambda_{\text {disp. }}^{2}
$$

In this case, it is not possible to measure $\Delta \lambda_{\text {disp from sky lines }}$ or arc lamps employed for wavelength calibration, because in both of those cases the source fills the entire slit. However, the angular size of the unresolved AGN point source is set by the seeing, which varies throughout the campaign but is almost always smaller than the $5^{\prime \prime}$ width of the slit. Our typical approach is therefore to search the literature for very highresolution measurements of the width of the [O III] lines, to serve as a measurement of $\Delta \lambda_{\text {true }}$, allowing $\Delta \lambda_{\text {disp }}$ to be determined. Such measurements do not exist for MCG-06-3015, but they do exist for NGC 1566, another Seyfert galaxy that we have monitored with the same instrument and setup.

For NGC 1566, Whittle (1992) measured $\mathrm{FWHM}=280 \mathrm{~km} \mathrm{~s}^{-1}$ for [O III] $\lambda 5007$ through a small slit, with a high resolution, and under good observing conditions. From our own spectra of NGC 1566 taken with RCSpec on the SMARTS $1.5 \mathrm{~m}$ telescope, we determined $\mathrm{FWHM}=8.26 \AA$ for [O III] $\lambda 5007$. We therefore deduce a value of $\Delta \lambda_{\text {disp }}=$ $6.8 \AA$ and adopt this value for our observations of MCG-06-3015. The final dispersion-corrected line widths and uncertainties for the mean and rms $\mathrm{H} \beta$ broad-line profiles are tabulated in Table 3.

\section{BLACK HOLE MASS}

The black hole mass is generally derived from reverberation mapping measurements as

$$
M_{\mathrm{BH}}=f \frac{R V^{2}}{G}
$$

where $R$ is taken to be $c \tau$, the speed of light times the mean time delay of a broad emission line relative to continuum variations, $V$ is the line-of-sight velocity of the gas in the BLR and is determined from the emission line width, and $G$ is the gravitational constant.

The factor $f$ is a scaling factor that accounts for the detailed geometry and kinematics of the gas in the BLR, and is generally unknown. In practice, it has become common to determine the population-average multiplicative factor, $\langle f\rangle$, necessary to bring the $M_{\mathrm{BH}}-\sigma_{\star}$ relationship for AGNs with 
reverberation masses into agreement with the $M_{\mathrm{BH}}-\sigma_{\star}$ relationship for nearby galaxies with dynamical black hole masses (e.g., Gültekin et al. 2009; Kormendy \& Ho 2013; McConnell \& Ma 2013). In this way, the overall scale for reverberation masses should be unbiased, but the mass of any single AGN is expected to be uncertain by a factor of 2-3. The value of $\langle f\rangle$ has varied in the literature from 5.5 (Onken et al. 2004a) to 2.8 (Graham et al. 2011), depending on which objects are included and the specifics of the measurements. We adopt the value determined by Grier et al. (2013) of $\langle f\rangle=4.3 \pm 1.1$.

Our preferred combination of measurements is $\tau_{\text {cent }}$ for the time delay and $\sigma_{\text {line }}$ measured from the rms spectrum for the line width. Combined with our adopted value of $\langle f\rangle$, we determine a black hole mass of $(1.6 \pm 0.4) \times 10^{6} M_{\odot}$ for MCG-06-30-15.

\section{DISCUSSION}

We present here the first optical emission-line reverberation results for MCG-06-30-15, but the well-studied nature of this AGN ensures that we have ample comparisons available in the literature with which we can assess our results. Lira et al. (2015) describe a long-term monitoring campaign in X-ray, optical, and near-IR bands from which several broad-band time delays were measured. In particular, they find that the near-IR bands lag the $B$ and $V$ bands by 13,20 , and 26 days in $J, H$, and $K$ respectively. While our monitoring campaign was not contemporaneous with that described by Lira et al. (2015), it was carried out the following observing season. Furthermore, Kara et al. (2014) find that the luminosity state of MCG-06-3015 did not change significantly over the period between 2001 and 2013, and the light curve from the Swift/BAT hard X-ray transient monitor shows no changes in luminosity state between 2005 and 2015 (Krimm et al. 2013). Comparison of our measured $\mathrm{H} \beta$ time delay of $\tau_{\text {cent }}=5.3 \pm 1.8$ days with the near-IR delays places the inner edge of the dust torus outside the BLR, as has been found for other Seyferts (Clavel et al. 1989; Suganuma et al. 2006; Koshida et al. 2014). Furthermore, our $\mathrm{H} \beta$ time delay compares remarkably well with the finding of Koshida et al. (2014) that $\tau(K) / \tau(\mathrm{H} \beta) \approx 4-5$. These findings are also in keeping with the scenario proposed by Netzer \& Laor (1993) in which the dust torus creates the outer edge of the BLR through suppression of line emission by the dust grains.

\subsection{Radius-Luminosity Relationship}

The empirical relationship between the BLR radius and the optical luminosity of the AGN (Kaspi et al. 2000, 2005; Bentz et al. 2006, 2009a, 2013) is a well-known scaling relationship derived from the set of reverberation mapping measurements for relatively nearby AGNs. The calibrated relationship relies on $\mathrm{H} \beta$ reverberation results and measurements of the continuum luminosity at $5100 \AA$, and it provides a quick way to estimate black hole masses without investing in time- and resource-intensive reverberation mapping programs for every target of interest. The $R_{\mathrm{BLR}}-L$ relationship has been found to be in good agreement with simple expectations from photoionization physics, once the luminosity measurements were corrected for the contribution from host-galaxy starlight measured through the spectroscopic aperture employed during the reverberation-mapping campaign (Bentz et al. 2006, 2009a, 2013). The scatter has also been found to be quite low, $<0.2$ dex (Bentz et al. 2013), implying that AGNs are mostly luminosity-scaled versions of each other.

Starlight corrections are especially important for nearby AGNs, such as MCG-06-30-15, because they can provide a significant fraction of the flux through the spectroscopic aperture. These corrections are generally obtained through two-dimensional surface brightness modeling of high-resolution images of AGN host galaxies. The decomposition allows the AGN PSF to be accurately separated from the host galaxy and the underlying sky, and thus an "AGN-free" image can be recovered from which the starlight flux can be measured. MCG-06-30-15 was observed with HST and the UVIS channel of WFC3 through the F547M filter as part of program GO-11662 to image the host galaxies of the LAMP 2008 AGN sample (Bentz et al. 2013). A single orbit was split into two pointings separated by a small-angle maneuver, and at each pointing a set of three exposures was taken, each exposure graduated in exposure time (short, medium, and long). The saturated pixels in the AGN core in the long exposures are corrected by scaling up the same pixels from the shorter, unsaturated exposures by the ratio of the exposure times. In this way, the graduated exposure times allow the dynamic range of the final drizzled image to significantly exceed the dynamic range of the detector itself. The total exposure time of the final combined, drizzled image is $2290 \mathrm{~s}$.

Two-dimensional surface brightness fitting of the HST image was carried out with the GALFIT software (Peng et al. 2002, 2010). We fit the host galaxy of MCG-06-30-15 with a Sérsic bulge and an exponential disk with an inner radial (truncation) function to approximate the dust lane. A single Fourier mode $(m=1)$ was also allowed for each of these components, to account for gross perturbations on the initial parametric models. The AGN and nearby star were fit with a model PSF generated by the Starfit algorithm (Hamilton 2014), which starts with a TinyTim PSF model (Krist 1993) and then fits the subpixel centering and the telescope focus. The underlying sky background was also fit as a gradient, and we used the entire field of view provided by WFC 3 to ensure that it was properly constrained, even though the galaxy itself covers only a small portion of the UVIS1 camera. The parameters for our best-fit model are tabulated in Table 4, and Figure 7 displays a region of the HST image centered on the galaxy (left), the best-fit model image (center), and the residuals after subtraction of the model from the image (right).

Using our best-fit model, we created a sky- and AGNsubtracted image of MCG-06-30-15. From this image, we measured the flux density of the host galaxy through the ground-based spectroscopic monitoring aperture (depicted as the white rectangle in the left panel of Figure 7). The scaling factor necessary to correct the flux density from the effective wavelength of the $H S T$ filter to $5100 \times(1+z)$ was determined with synphot and a template spectrum of a galactic bulge (Kinney et al. 1996). Our determination of the flux density of the host galaxy at $5100 \times(1+z)$ is $f_{\text {gal }}=(4.28 \pm 0.43) \times$ $10^{-15} \mathrm{erg} \mathrm{s}^{-1} \mathrm{~cm}^{-1} \AA^{-1}$. The average flux density at $5100 \times(1+z)$ was determined from our scaled spectra to be $f_{\text {obs }}=(5.23 \pm 0.08) \times 10^{-15} \mathrm{erg} \mathrm{s}^{-1} \mathrm{~cm}^{-1} \AA^{-1}$. Correcting for the host-galaxy contribution, we deduce an AGN-only flux density of $f_{\mathrm{AGN}}=(0.95 \pm 0.49) \times 10^{-15} \mathrm{erg} \mathrm{s}^{-1} \mathrm{~cm}^{-1} \AA^{-1}$.

Unfortunately, the distance to MCG-06-30-15 is not particularly well constrained. The luminosity distance implied by the galaxy redshift is $D_{L}=32.5 \mathrm{Mpc}$. However, the 
Table 4

Surface Brightness Decomposition

\begin{tabular}{|c|c|c|c|c|c|c|c|c|c|}
\hline \multirow[t]{5}{*}{$\#$} & PSF+sky & $\Delta x\left({ }^{\prime \prime}\right)$ & $\Delta y\left({ }^{\prime \prime}\right)$ & $m_{\text {stmag }}{ }^{\mathrm{a}}$ & $\ldots$ & Sky (counts) & $\frac{d \text { sky }}{d x}\left(10^{-4}\right.$ counts $)$ & $\frac{d \text { dsky }}{d y}\left(10^{-4}\right.$ counts $)$ & Note \\
\hline & Sersic & $\Delta x\left({ }^{\prime \prime}\right)$ & $\Delta y(")$ & $m_{\text {stmag }}$ & $r_{\mathrm{e}}\left({ }^{\prime \prime}\right)$ & $n$ & $b / a$ & PA (deg) & \\
\hline & Sersic 3 & $\Delta x\left({ }^{\prime \prime}\right)$ & $\Delta y\left({ }^{\prime \prime}\right)$ & $\Sigma_{\text {stmag }}$ & $r_{\mathrm{e}}\left({ }^{\prime \prime}\right)$ & $n$ & $b / a$ & PA (deg) & \\
\hline & Radial & $\Delta x\left({ }^{\prime \prime}\right)$ & $\Delta y\left({ }^{\prime \prime}\right)$ & $\ldots$ & $r_{\text {break }}\left({ }^{\prime \prime}\right)$ & $\Delta r_{\text {soft }}\left({ }^{\prime \prime}\right)$ & $b / a$ & PA (deg) & \\
\hline & Fourier & $\cdots$ & mode: $a$ & $b(\operatorname{deg})$ & $\ldots$ & $\ldots$ & $\cdots$ & $\cdots$ & \\
\hline (1) & (2) & (3) & (4) & (5) & (6) & (7) & (8) & (9) & (10) \\
\hline 1,2 & $\mathrm{PSF}+\mathrm{sky}$ & 0.000 & 0.000 & 16.26 & $\cdots$ & 31.72 & -3.7 & 4.2 & \\
\hline \multirow[t]{2}{*}{3} & Sersic & 0.038 & -0.008 & 15.70 & 1.014 & 1.9 & 0.47 & -36.8 & bulge \\
\hline & Fourier & 1: -0.418 & -96.2 & & & & & & \\
\hline \multirow[t]{3}{*}{4} & Sersic3 & 0.132 & -0.092 & 18.66 & 8.216 & {$[1.0]$} & 0.48 & -32.1 & disk \\
\hline & Radial,inner & 1.036 & 0.764 & $\ldots$ & 2.149 & 2.195 & 0.35 & -24.7 & dust lane \\
\hline & Fourier & 1: 0.722 & 116.5 & & & & & & \\
\hline Merit & & $\chi^{2}=6453$ & 72.0 & & $N_{\mathrm{dof}}=15$ & 3039 & $N_{\text {free }}=29$ & $\chi_{\nu}^{2}=40.88$ & \\
\hline
\end{tabular}

Notes. Values in square brackets were held fixed when fitting the surface brightness model.

${ }^{a}$ The STmag magnitude system is based on the absolute physical flux per unit wavelength.
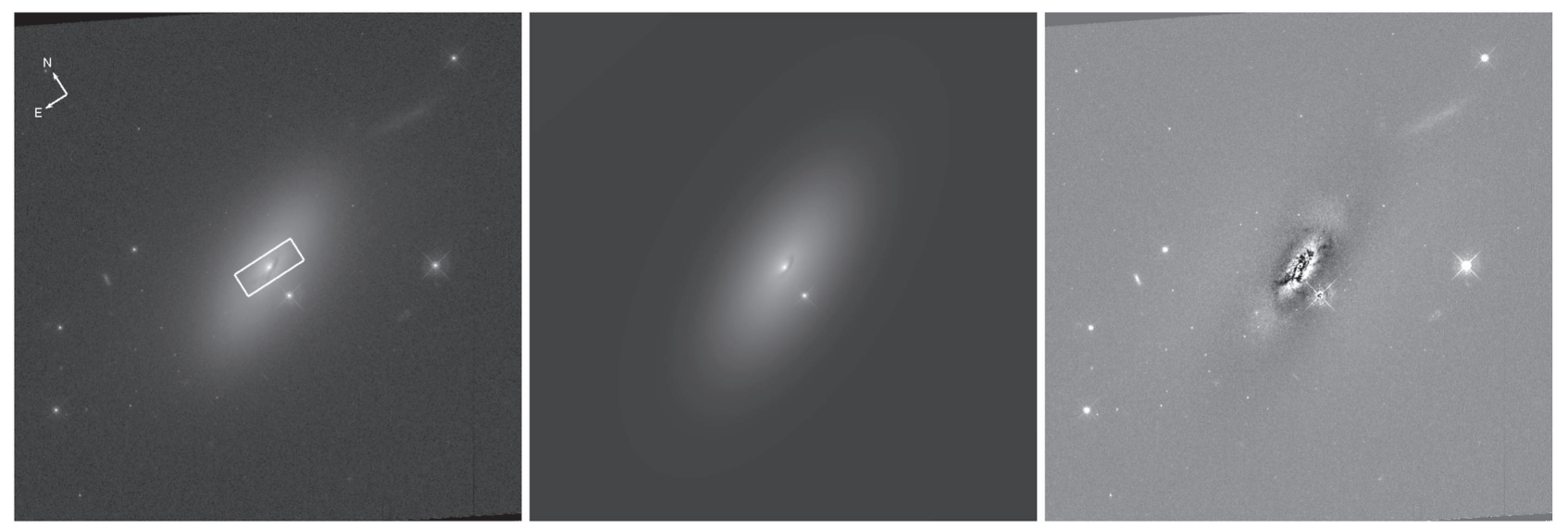

Figure 7. Left: $H S T$ image of the host galaxy of MCG-06-30-15, displayed with a logarithmic stretch. The area shown is $80^{\prime \prime} \times 80^{\prime \prime}$ and is only a portion of the full field of view. The white rectangle centered on the nucleus of the galaxy shows the ground-based spectroscopic monitoring aperture. Middle: GALFIT model, displayed with the same stretch as the image. Right: residuals after subtracting the model from the image, displayed with a linear stretch centered around zero counts.

Extragalactic Distance Database (Tully et al. 2009) reports $D=25.5 \pm 3.5 \mathrm{Mpc}$ from their model of cosmic flows and the group membership of MCG-06-30-15 (Tully et al. 2013). Taken at face value, this $\sim 30 \%$ disagreement in distance leads to an uncertainty by a factor of 1.6 in the luminosity. Additionally, there are only three galaxies contributing to the determination of the group distance, and the individual distance estimates for these three galaxies range from 20 to $36 \mathrm{Mpc}$. As part of a separate program to determine distances to AGN host galaxies using the Tully-Fisher method (Tully \& Fisher 1977), we observed MCG-06-30-15 with the Green Bank Telescope, but we were unable to detect $\mathrm{H} \mathrm{I} 21 \mathrm{~cm}$ emission with $3.5 \mathrm{hr}$ of on-source time. For our purposes here, we adopt the estimate from cosmic flows and its uncertainty, but we note that it will be important to better constrain the distance to this galaxy in order to determine more accurate physical parameters (including, but not limited to, any luminosity measurements). After correcting for Galactic extinction along the line of sight as determined by Schlafly \& Finkbeiner (2011), we find $\log \lambda L_{\lambda}(5100 \AA)=41.65 \pm 0.25 \mathrm{erg} \mathrm{s}^{-1}$.

Figure 8 depicts the location of MCG-06-30-15 on the $R_{\mathrm{BLR}}-L$ relationship. We have not determined a new best fit to the relationship, but have simply recreated the plot from Bentz et al. (2013). MCG-06-30-15 is fairly consistent with the typical scatter around the relationship. We note that if we were to adopt one of the other measurements of time delay (such as $\tau_{\text {jav }}$ ), or the luminosity distance from the galactic redshift, the agreement would be even better. With the adopted assumptions, and furthermore assuming that $L_{\mathrm{bol}}=4.9+0.9 \lambda L_{\lambda}(5100 \AA$ ) (Runnoe et al. 2012), we estimate $L / L_{\mathrm{Edd}}=0.04$.

\subsection{Consistency of the Black Hole Mass}

Our measurement of $M_{\mathrm{BH}}=(1.6 \pm 0.4) \times 10^{6} M_{\odot}$ for MCG-06-30-15 is in excellent agreement with the value determined by McHardy et al. (2005) of $M_{\mathrm{BH}}=2.9_{-1.6}^{+1.8} \times$ $10^{6} M_{\odot}$. Their work assumed a linear scaling between $M_{\mathrm{BH}}$ and the break in the X-ray power spectral density, with the relationship anchored to the measurements for the Galactic black hole Cygnus X-1. This agreement therefore bolsters the claim that supermassive black holes are simply analogs of Galactic black holes, but scaled up in mass (e.g., McHardy et al. 2006).

Raimundo et al. (2013) describe integral field spectroscopic observations using VLT SINFONI of the innermost $\sim 0.5 \mathrm{kpc}$ of the galaxy in the $H$ band. Although their observations were somewhat shallow (total on-source exposure time of $1.3 \mathrm{hr}$ ), they attempted to constrain the black hole mass with the Jeans 


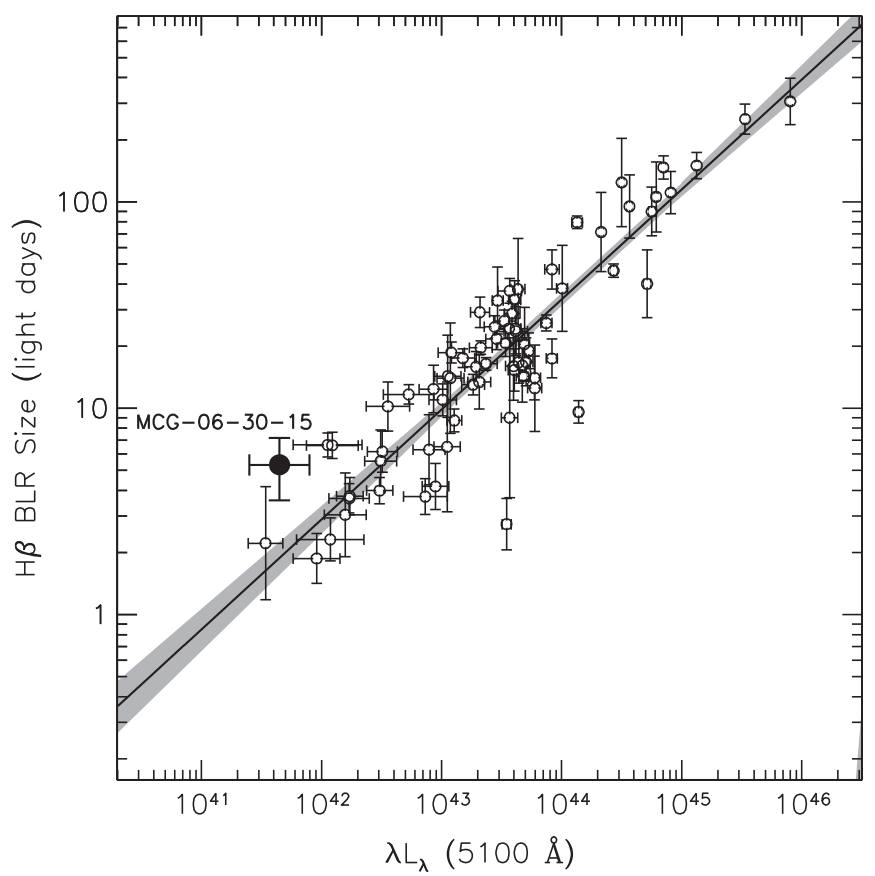

Figure 8. Radius-luminosity relationship for AGNs (open points and fit; Bentz et al. 2013) with the $\mathrm{H} \beta$ time delay and AGN luminosity for MCG-06-30-15 from this work plotted as the filled point.

anisotropic modeling (Cappellari 2008). Intriguingly, they find a best-fit value of $M_{\mathrm{BH}}=4 \times 10^{6} \quad M_{\odot}$ (assuming $D=37 \mathrm{Mpc}$ ), although they caution that there is actually a stronger constraint on an upper limit of $M_{\mathrm{BH}}<6 \times 10^{7} M_{\odot}$ than on the best-fit mass.

One of our original reasons for targeting MCG-06-30-15 was the fact that it might be possible to determine a black hole mass through both reverberation mapping and stellar dynamical modeling for this nearby AGN. The sample of objects for which we are able to compare these two methods of mass determination is extremely small for two reasons: (1) stellar dynamical modeling is limited by spatial resolution, and therefore distance; and (2) broad-lined AGNs in the local universe are quite rare, and therefore generally far away. Only two galaxies have published masses from both methods thus far -NGC 4151 (Bentz et al. 2006; Onken et al. 2014b) and NGC 3227 (Davies et al. 2006; Denney et al. 2010).

A useful metric for determining whether a stellar dynamical mass is likely to be achievable is to determine whether the black hole sphere of influence $\left(r_{h}\right)$ could be resolved with the observations, where

$$
r_{h}=\frac{G M_{\mathrm{BH}}}{\sigma_{\star}^{2}} .
$$

Combining our mass with the value of $\sigma_{\star}=109 \mathrm{~km} \mathrm{~s}^{-1}$ determined by Raimundo et al. (2013) and the distance of $25.5 \mathrm{Mpc}$ adopted above, we estimate $r_{h}=0$."005. This scale is not resolvable with currently available instruments, although Gültekin et al. (2009) argue that it is not strictly necessary to resolve $r_{h}$ to obtain a useful constraint on $M_{\mathrm{BH}}$. Furthermore, the best-fit black hole mass derived by Raimundo et al. (2013), even with shallow observations and a spatial scale of 0 ". 05 , suggests that it could be worthwhile to pursue a stellar dynamical mass constraint for MCG-06-30-15. In this case, an accurate distance will be even more necessary, because dynamical masses scale linearly with the assumed distance.

Time lags between different X-ray energy bands have also been detected in MCG-06-30-15 (Emmanoulopoulos et al. 2011; De Marco et al. 2013; Kara et al. 2014). Of particular interest are soft X-ray lags (where low-energy X-rays lag behind higher-energy X-rays), likely due to X-ray reverberation (Fabian et al. 2009). Emmanoulopoulos et al. (2011) first detected a soft lag of approximately $20 \mathrm{~s}$ in MCG06-30-15. A systematic search for, and analysis of, soft lags in X-ray-variable AGNs found that the amplitude of the soft lags and the Fourier frequency where they are observed scale with black hole mass (De Marco et al. 2013). MCG-06-30-15 is one of the 15 detections of soft lags used to determine the scaling relation, with a black hole mass estimated from the $R_{\mathrm{BLR}}-L$ relationship. Ignoring the fact that MCG-06-30-15 was used to determine the scaling relation for soft lags, and that the scaling relation is subject to selection biases (De Marco et al. 2013), we can use the soft lag to estimate the black hole mass. De Marco et al. (2013) measure a soft lag of $26.4 \pm 12.7 \mathrm{~s}$, which predicts a black hole mass of $1.1 \times 10^{6} M_{\odot}$, consistent with the reverberation mass we have determined in this work.

\subsection{M- $\sigma$ Relationship for AGNs}

We also examine the black hole mass we have derived for MCG-06-30-15 in light of the $M_{\mathrm{BH}}-\sigma_{\star}$ relationship for other AGNs with reverberation masses. Raimundo et al. (2013) constrained the stellar velocity dispersion of the bulge using a pseudoslit geometry and velocity dispersion maps obtained with VLT SINFONI and they determined $\sigma_{\star}=109 \mathrm{~km} \mathrm{~s}^{-1}$. This value is somewhat larger than the value of $\sigma_{\star}=93.5 \pm 8.5 \mathrm{~km} \mathrm{~s}^{-1}$ determined by McHardy et al. (2006) from long-slit spectroscopy.

In Figure 9, we show the $M_{\mathrm{BH}}-\sigma_{\star}$ relationship for AGNs from Grier et al. (2013). MCG-06-30-15 sits a bit below and to the right of the relationship, but appears to be fairly consistent within the scatter. Adoption of the value of $\sigma_{\star}$ from McHardy et al. (2006) would further bolster the agreement.

We note that we have an independent project currently in progress that will recalibrate the $M_{\mathrm{BH}}-\sigma_{\star}$ relationship for AGNs using velocity dispersions derived solely from integral field spectroscopy; this will be important for removing any effects of rotational broadening from the $\sigma_{\star}$ measurements among the rest of the sample (Batiste \& Bentz 2016), as well as any scatter imposed by the selection of a specific position angle for long-slit observations. We intend to revisit the location of MCG-06-30-15 on this relationship at that time.

\section{SUMMARY}

We have determined a reverberation time delay for the broad $\mathrm{H} \beta$ emission line in the spectrum of MGC-06-30-15 of $\tau_{\text {cent }}=5.3 \pm 1.8$ days in the rest frame of the AGN. The measured time delay is in good agreement with the $R_{\mathrm{BLR}}-L$ relationship for AGNs. It also agrees with the relationship between $\mathrm{H} \beta$ and near-IR time delays, where the effective optical size of the BLR is approximately 4-5 times smaller than the inner edge of the dust torus. Combining the $\mathrm{H} \beta$ measurement of time delay with the width of the emission 


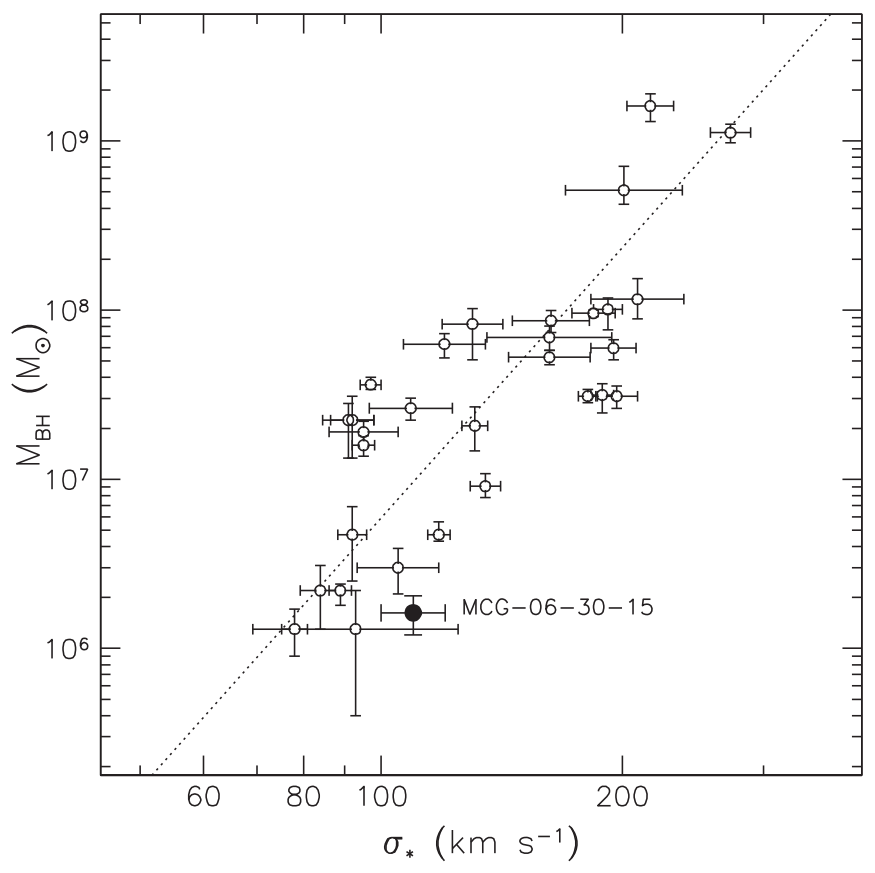

Figure 9. $M_{\mathrm{BH}^{-}}-\sigma_{\star}$ relationship for AGNs (open points and fit; Grier et al. 2013) with MCG-06-30-15 plotted as the filled point.

line in the variable part of the spectrum, we constrain a central black hole mass of $M_{\mathrm{BH}}=(1.6 \pm 0.4) \times 10^{6} M_{\odot}$. This value is in good agreement with estimates from the break in the X-ray power spectral density and $M_{\mathrm{BH}}-\sigma_{\star}$ relationships.

We thank the referee for comments that improved the presentation of this paper. M.C.B. gratefully acknowledges support from the NSF through CAREER grant AST-1253702. K.H. acknowledges support from UK Science and Technology Facilities Council (STFC) grant ST/M001296/1. We thank the SMARTS Consortium members and CTIO staff for efforts to make observations at the CTIO/SMARTS $1.5 \mathrm{~m}$ telescope possible. This work makes use of observations from the LCOGT network and is based on observations with the NASA/ESA Hubble Space Telescope. We are grateful for support of this work through grant HST GO-11662 from the Space Telescope Science Institute, which is operated by the Association of Universities for Research in Astronomy, Inc., under NASA contract NAS5-26555. The Liverpool Telescope is operated on the island of La Palma by Liverpool John Moores University in the Spanish Observatorio del Roque de los Muchachos of the Instituto de Astrofisica de Canarias with financial support from the UK Science and Technology Facilities Council. This research has made use of the NASA/ IPAC Extragalactic Database (NED), which is operated by the Jet Propulsion Laboratory, California Institute of Technology, under contract with the National Aeronautics and Space Administration, and the SIMBAD database, operated at CDS, Strasbourg, France.

\section{REFERENCES}

Alard, C. 2000, A\&AS, 144, 363

Alard, C., \& Lupton, R. H. 1998, ApJ, 503, 325

Antonucci, R. 1993, ARA\&A, 31, 473

Batiste, M., \& Bentz, M. C. 2016, in American Astronomical Society Meeting 227, Abstract 104.07
Bentz, M. C., Denney, K. D., Grier, C. J., et al. 2013, ApJ, 767, 149

Bentz, M. C., Peterson, B. M., Netzer, H., Pogge, R. W., \& Vestergaard, M. 2009a, ApJ, 697, 160

Bentz, M. C., Peterson, B. M., Pogge, R. W., Vestergaard, M., \& Onken, C. A. 2006, ApJ, 644, 133

Bentz, M. C., Walsh, J. L., Barth, A. J., et al. 2008, ApJL, 689, L21

Bentz, M. C., Walsh, J. L., Barth, A. J., et al. 2009b, ApJ, 705, 199

Blandford, R. D., \& McKee, C. F. 1982, ApJ, 255, 419

Brenneman, L. W., \& Reynolds, C. S. 2006, ApJ, 652, 1028

Cappellari, M. 2008, MNRAS, 390, 71

Chiang, C.-Y., \& Fabian, A. C. 2011, MNRAS, 414, 2345

Clavel, J., Wamsteker, W., \& Glass, I. S. 1989, ApJ, 337, 236

Davies, R. I., Thomas, J., Genzel, R., et al. 2006, ApJ, 646, 754

De Marco, B., Ponti, G., Cappi, M., et al. 2013, MNRAS, 431, 2441

Denney, K. D., Peterson, B. M., Pogge, R. W., et al. 2010, ApJ, 721, 715

Edelson, R., Turner, T. J., Pounds, K., et al. 2002, ApJ, 568, 610

Emmanoulopoulos, D., McHardy, I. M., \& Papadakis, I. E. 2011, MNRAS, 416, L94

Fabian, A. C. 2012, ARA\&A, 50, 455

Fabian, A. C., Zoghbi, A., Ross, R. R., et al. 2009, Natur, 459, 540

Fath, E. A. 1913, ApJ, 37, 198

Ferrarese, L., \& Ford, H. 2005, SSRv, 116, 523

Ferrarese, L., \& Merritt, D. 2000, ApJL, 539, L9

Gaskell, C. M., \& Peterson, B. M. 1987, ApJS, 65, 1

Gaskell, C. M., \& Sparke, L. S. 1986, ApJ, 305, 175

Gebhardt, K., Bender, R., Bower, G., et al. 2000, ApJL, 539, L13

Graham, A. W., Onken, C. A., Athanassoula, E., \& Combes, F. 2011, MNRAS, 412, 2211

Grier, C. J., Martini, P., Watson, L. C., et al. 2013, ApJ, 773, 90

Grier, C. J., Peterson, B. M., Pogge, R. W., et al. 2012, ApJ, 755, 60

Gültekin, K., Richstone, D. O., Gebhardt, K., et al. 2009, ApJ, 698, 198

Hamilton, T. S. 2014, in American Astronomical Society Meeting 223, Abstract 145.02

Heckman, T. M., \& Best, P. N. 2014, ARA\&A, 52, 589

Kara, E., Fabian, A. C., Marinucci, A., et al. 2014, MNRAS, 445, 56

Kaspi, S., Maoz, D., Netzer, H., et al. 2005, ApJ, 629, 61

Kaspi, S., Smith, P. S., Netzer, H., et al. 2000, ApJ, 533, 631

King, A., \& Pounds, K. 2015, ARA\&A, 53, 115

Kinney, A. L., Calzetti, D., Bohlin, R. C., et al. 1996, ApJ, 467, 38

Koleva, M., Prugniel, P., Bouchard, A., \& Wu, Y. 2009, A\&A, 501, 1269

Kormendy, J., \& Ho, L. C. 2013, ARA\&A, 51, 511

Koshida, S., Minezaki, T., Yoshii, Y., et al. 2014, ApJ, 788, 159

Krawczynski, H., \& Treister, E. 2013, FrPhy, 8, 609

Krimm, H. A., Holland, S. T., Corbet, R. H. D., et al. 2013, ApJS, 209, 14

Krist, J. 1993, in ASP Conf. Ser. 52, Astronomical Data Analysis Software and Systems II (San Francisco, CA: ASP), 536

Lira, P., Arévalo, P., Uttley, P., McHardy, I. M. M., \& Videla, L. 2015, MNRAS, 454, 368

Magorrian, J., Tremaine, S., Richstone, D., et al. 1998, AJ, 115, 2285

Marinucci, A., Matt, G., Miniutti, G., et al. 2014, ApJ, 787, 83

McConnell, N. J., \& Ma, C.-P. 2013, ApJ, 764, 184

McHardy, I. M., Gunn, K. F., Uttley, P., \& Goad, M. R. 2005, MNRAS, 359, 1469

McHardy, I. M., Koerding, E., Knigge, C., Uttley, P., \& Fender, R. P. 2006, Natur, 444, 730

Morris, S. L., \& Ward, M. J. 1988, MNRAS, 230, 639

Netzer, H. 2015, ARA\&A, 53, 365

Netzer, H., \& Laor, A. 1993, ApJL, 404, L51

Onken, C. A., Ferrarese, L., Merritt, D., et al. 2004a, ApJ, 615, 645

Onken, C. A., Valluri, M., Brown, J. S., et al. 2014b, ApJ, 791, 37

Peng, C. Y., Ho, L. C., Impey, C. D., \& Rix, H. 2002, AJ, 124, 266

Peng, C. Y., Ho, L. C., Impey, C. D., \& Rix, H.-W. 2010, AJ, 139, 2097

Peterson, B. M. 1993, PASP, 105, 247

Peterson, B. M., Berlind, P., Bertram, R., et al. 2002, ApJ, 581, 197

Peterson, B. M., Ferrarese, L., Gilbert, K. M., et al. 2004, ApJ, 613, 682

Peterson, B. M., Wanders, I., Bertram, R., et al. 1998a, ApJ, 501, 82

Peterson, B. M., Wanders, I., Horne, K., et al. 1998b, PASP, 110, 660

Raimundo, S. I., Davies, R. I., Gandhi, P., et al. 2013, MNRAS, 431, 2294

Rees, M. J. 1984, ARA\&A, 22, 471

Reynolds, C. S., Ward, M. J., Fabian, A. C., \& Celotti, A. 1997, MNRAS, 291, 403

Rodríguez-Pascual, P. M., Alloin, D., Clavel, J., et al. 1997, ApJS, 110, 9

Runnoe, J. C., Brotherton, M. S., \& Shang, Z. 2012, MNRAS, 422, 478

Schlafly, E. F., \& Finkbeiner, D. P. 2011, ApJ, 737, 103

Siverd, R. J., Beatty, T. G., Pepper, J., et al. 2012, ApJ, 761, 123

Storey, P. J., \& Zeippen, C. J. 2000, MNRAS, 312, 813 
Suganuma, M., Yoshii, Y., Kobayashi, Y., et al. 2006, ApJ, 639, 46 Tanaka, Y., Nandra, K., Fabian, A. C., et al. 1995, Natur, 375, 659

Tully, R. B., Courtois, H. M., Dolphin, A. E., et al. 2013, AJ, 146, 86 Tully, R. B., \& Fisher, J. R. 1977, A\&A, 54, 661

Tully, R. B., Rizzi, L., Shaya, E. J., et al. 2009, AJ, 138, 323

Urry, C. M., \& Padovani, P. 1995, PASP, 107, 803

van Groningen, E., \& Wanders, I. 1992, PASP, 104, 700
Vazdekis, A., Sánchez-Blázquez, P., Falcón-Barroso, J., et al. 2010, MNRAS, 404, 1639

Walsh, J. L., Minezaki, T., Bentz, M. C., et al. 2009, ApJS, 185, 156

White, R. J., \& Peterson, B. M. 1994, PASP, 106, 879

Whittle, M. 1992, ApJS, 79, 49

Winkler, H. 1992, MNRAS, 257, 677

Zu, Y., Kochanek, C. S., \& Peterson, B. M. 2011, ApJ, 735, 80 